\title{
A DECISION ANALYSIS MAINTENANCE MODEL (SYSTEM): AN APPLICATION OF A.H.P. AND A FUZZY LOGIC RULE-BASED CONTROLLER.
}

\author{
Ashraf W. Labib, Glyn B. Williams and Richard F. O'Connor \\ Integrated Logistic Management Group, \\ School of Manufacturing \& Mechanical Engineering, \\ University of Birmingham, B15 2TT. U.K. \\ a.labib@bham.ac.uk
}

\begin{abstract}
In this paper, an attempt to develop an integrated model of Maintenance Decision Making by utilising AHP and FUZZY LOGIC is presented. The paper describes problems in maintenance arising from not having clear criteria and not having robust decision with which to maintain failing equipment. A two step process is proposed. The first step is to obtain a prioritised criteria for maintenance and hence most critical machines and their related faults using AHP analysis. The second step use the weights obtained from the first step as crisp inputs to a fuzzy logic controller in order to obtain a prescriptive model for maintenance action.
\end{abstract}

\section{Introduction}

One of the major problems in maintenance is the lack of a systematically, focused and adaptable approach in setting preventive maintenance instructions. Hence, preventive maintenance instructions tend to be static, and are neither efficient nor effective. This paper is concerned with the operational aspects of maintenance in terms of machine criticality and fault analysis. The paper is divided into two integrated models; a multiple criteria decision making (MCDM_Maintenance) model and, a fuzzy logic rule-based (FLRB_Maintenance) model. The MCDM model seeks effectiveness through prioritising machines' criticality, and focusing on specific components that will maximise gains based on different criteria. The extended model (the FLRB system) seeks an efficient approach to specify the most appropriate maintenance action to follow based on different rules. Therefore, in the first model (MCDM Maintenance System) the AHP is used to prioritise machines and their faults, while in the second model (Fuzzy Logic Rule-Base system) the rule-base based on fuzzy logic is used to determine the most efficient actions to be undertaken to overcome these faults. Therefore the output of the former model is considered as an input to the latter one. In short, the MCDM_Maintenance system attempts to answer the "WHAT" type of questions (an effectiveness measure), whereas, the Fuzzy Logic Rule-Base (FLRB) system answers the "HOW" type of questions (an efficiency) measure.

The system developed can handle multiple criteria decision analysis, and subjective judgements. Moreover, the methodology facilitates and supports a group decision making process. This systematic, and adaptable, approach will determine what specific actions to perform given current working conditions. The methodology employed in this system enables it to be integrated into any historical data found in computerised maintenance management systems. This approach can also be adapted to act as a real-time feedback mechanism for setting priorities in a condition monitoring environment. The proposed system works in four stages. The first stage involves identifying the criteria upon which engineering personnel wish to formulate a maintenance decision, or action. Once criteria are identified, the second stage is to prioritise the different criteria by implementing a multiple criteria evaluation method. The evaluation is carried out in a structured approach using the Analytic Hierarchy Process (AHP) methodology. The third stage involves ranking machines according to criticality based on different criteria. This is followed by an in-depth detailed and focused analysis of failures in a graphical and a hierarchical format. Finally, a fuzzy logic rule-based controller is used to identify most efficient action to be undertaken based on different rules. This final stage is further divided into three steps of fuzzification, rules evaluation, and defuzzification.

\section{Background And Motivation For Development Of An Integrated Maintenance Model}

\section{Background}

Maintenance as a function, compared to other areas in operations, is considered to be of a fuzzy nature. Maintenance has not led itself to systemisation due to the fact that its activities were not repetitive in the same manner as operations tasks. Hence, there is a need for an iterative and a systematic approach to maintenance practice. 
It has been observed that decision makers in maintenance often seek to be efficient before being effective. As an example, the maintenance manager out of his/her concern to minimise the workload pressure, and resources, will tend to prioritise the worst machines based on the number of maintenance calls. Whereas, other criteria might be of similar, or even of more, importance. Examples of other important criteria can be downtime, capacity and bottle-neck constraints, and machines' spare parts cost. It has also been observed that, in practice, both preventive and corrective maintenance belong to two separate worlds. Although preventive maintenance is usually initiated as an outcome to prevent unplanned breakdowns from occurring. It is often observed that as time passes and conditions change, breakdown types differ in their duration, frequency and effect, whereas, old preventive instructions remain the same and lack adaptability and flexibility. Furthermore, to achieve economy of scale in carrying out preventive maintenance, preventive instructions tend to be standardised in their form and timing. This approach, of applying standard wholesale, instructions might be counter productive and the effectiveness of such an approach could be difficult to quantify. It then becomes a case of "wait and see" whether the cost spent on preventive maintenance is more (or less) than breakdowns occurring and then adjust accordingly. Hence, a more efficient approach is needed. This approach should be able to control preventive maintenance strategies based on an adaptable mechanism. This mechanism should be able to identify most effective areas where maximum benefits are expected.

Mathematical models have been formulated for many typical situations. These models can be useful in answering questions such as: How much maintenance should be done on this machine? How frequently should this part be replaced? How many spares should be kept in stock? How should the shutdown be scheduled? Emphasis by [Vanneste and Wassenhove, 1995], [Kobbacy et al., 1995], and [Cho and Parlar, 1991] have shown that the vast majority of maintenance models are aimed at answering efficiency questions, that is questions of the form "how can this particular machine be operated more efficiently?", and not at effectiveness questions, like "which machine should we improve and how?". The latter question is often the one in which practitioners are interested. From this perspective it is not surprising that practitioners are often dissatisfied if a model is directly applied to an isolated problem. This is precisely why in the integrated approach to efficiency analysis as proposed by the authors (do the thing right) is preceded by effectiveness analysis (do the right thing).

\section{Motivation}

A Multiple Criteria Decision Making (MCDM) Maintenance model has been developed, and has been partially verified, as it is currently being tested for implementation in industry. The motivation for developing this MCDM Maintenance model is three-fold. First, it shows a practical implementation of the traditional TPM (Total Productive Maintenance) model. This is due to the fact that the proposed model addresses the three axioms of TPM; equipment effectiveness, autonomous maintenance, and small group activities in an innovative model based on a computerised multiple criteria decision making approach. The second motivation is that there is evidence in that current practice in maintenance decisions are not yet effective. This evidence is based on the fact that only $2 \%$ of information collected is ever used for decision analysis, while most of the time is used in data collection and data analysis [Dyer, and Forman, 1991]: In addition, evidence from research [McKinnon, 1992] conducted since mid - 1960s shows that most managers do not rely on computer-based information to make decisions. The final motivation is that the developed model (system) offers an opportunity to analyse and validate industrial data in a systematic, a graphical and, a hierarchical approach.

The design of the model (system) was based on four principles. The first principle is to seek effectiveness before efficiency [Vanneste, And Wassenhove, 1995]. The approach consists of two major parts. The first part is effectiveness analysis. The aim of effectiveness analysis is to detect the most important problems and potential solutions. Once the potential solutions to the most important problems are identified and prioritised it becomes clear which task or procedure has to be carried out more efficiently. To reach maximum efficiency a further analysis of the given task or procedure is necessary. This is the second part, the efficiency analysis. This approach shows why using AHP to prioritise (effectiveness), followed by optimising the solutions for the potential problems (efficiency), is an appropriate approach. While effectiveness is concerned with 'doing the right thing', efficiency aims at 'doing the thing right'. Thus, effectiveness analysis ensures that the effort put in improving efficiency is indeed dedicated to important tasks. Hence, maximum value added benefit is achieved. The second principle is to formulate the problem in a hierarchical structure that is composed of a general upper level of objectives, or criteria, and lower levels of specific critical machines and fault details. This principle is based on the structural formulation of the AHP model [Saaty, and Vargas, 1994] as a multiple criteria decision making approach. The AHP was used since, as stated by [Saaty, 1990b], "it is a decision theory that directly interprets data and information by forming judgements and performing ratio scale measurement in them within a prescribed hierarghical framework". The third principle is to utilise 
existing experience, or data, as an aid for taking decisions. This "experience-based" approach was presented by [Laakso, etal, 1995] in a Reliability Centred Maintenance (RCM) context. Hence, the model (system) is connected to an existing reporting system that contains data on failure events and the chronology of failure actions and related reports. However, the developed system can be connected to any computerised environment, as long as there is history of what needs to be prioritised in terms of criteria or alternatives. An example of other environments can be condition monitoring where the system (model) can be adapted to act as a feedback mechanism for setting priorities in a condition monitoring environment. The last principle is based on the fact that decision tools should allow, not only quantitative absolute measures, but also qualitative ones. To be realistic, models have to include and measure all important quantitative and qualitative factors. In this work, AHP provides the framework and technique in a way that satisfies this tenet. As an example, the problem solver is given the flexibility to trade increased productivity, with decreasing quality defects, and increasing machines' availability. To the knowledge of the authors, this approach of identifying criticality of machines and fault details in an interactive multiple criteria method has not been developed yet.

The motivation for developing the FLRB-Maintenance model is four-fold. First, reaching the most appropriate strategy requires an efficient, simple, and workable tool. It is generally known that the goal of any control system is to produce a desired set of outputs for a given set of inputs. A common solution to these sort of problems is to have a controller that executes a mathematical formula that expresses the output as a function of the input. Theoretically, the formula represents an accurate model of the system behaviour. In practice, however, formulas can be quite complex. Working them out in real time may not be feasible. In addition, it may be difficult, or even impossible, to derive a workable mathematical model in the first place. For example, an automotive engineer might understand the general relationship between, say, ignition timing, air flow, fuel mix, and engine rev./min., but exact mathematics underlying those relationships may be completely unclear. The same example can be applied to the general maintenance problem, where the relationship among different components and their faults might be attributed to different factors such as frequency of maintenance calls, downtime, skill levels, spare parts cost, and others. Rule-based fuzzy controllers do not require complex mathematical models. All that is really needed is a practical understanding of the overall system behaviour. The second motivation is that when controlling a complex system, it is necessary to attribute the behaviour of the system to different set of parameters, not just one. This was nicely described by the behavioural scientist Abraham Maslow as follows" when the only tool a person has is a hammer, he'll treat everything else as a nail". Thus, when dealing with choosing the most efficient maintenance action to follow for a specific machine, or component, it is important to examine the effect of at least more than one factor, through developing sets of rules based on expert knowledge. These rules describe the output of the system (in our case maintenance action), based on the combination of inputs (in our case frequency and downtime). The third motivation is related to the fact that although the MCDM_Maintenance system based on the AHP is powerful in its ability to focus on identifying the major faults of the most problematic machines based on different criteria, the prioritisation is not global. It is quite impossible to consider all faults that are related to all machines which are related to all criteria at the same time to arrive to a global solution. This is a common problem in other issues related to maintenance such as machines' release and scheduling. This complexity is because they are combinatorial problems to which no optimisation approach can be applied efficiently [Hintz, and Zimmermann, 1989]. However, integrating a fuzzy rule-base controller to the AHP process can help in deriving the most efficient action to follow based on the effect of different parameters, and hence, insuring a "near optimum solution". The final motivation is that the MCDM_Maintenance system developed using AHP offers the decision maker a broader picture of the problem, by examining it from different angles (criteria). Therefore, when running the system on the same set of data several times the "sense" of scale of the total picture becomes clear. This feature is of importance in the linkage to the fuzzy logic controller, as the perception of what is considered to be "small", "medium" and "high" values of inputs needs to be identified in the fuzzifying process as will be shown later in this paper. The flow of the integrated model is shown in Fig (1). Notice that inputs to the model are data from any computerised maintenance management system (CMMS), and outputs are decisions. The transformation of data to decisions is a core concept, and motivation for the development of the model.

The integration of AHP and fuzzy logic is found to be beneficial in a number of aspects. The AHP primary function is to prioritise machines and their faults based on different criteria, and hence a criticality output is produced. A secondary function of AHP is modelling the problem from different perspectives (angles). This function offers the decision maker a "sense" of the scale of the problem. Both functions; priority and scale, are of great importance when using fuzzy logic. The priorities in terms of frequency, or downtime, or any other criteria, are crisp values to the fuzzy logic process. Scaling produced by the AHP when comparing any fault, and/or machine, with others gives an indication of what values can be considered as low, medium, or high. The ability of fuzzy logic to utilise IF - THEN rules offers an interaction of the expertise of the decision maker to react to the analysis offered by the AHP. The decision maker can either alter the rules, or the scaling values. 
The model is considered integrated and adaptable in the sense that it offers the decision maker the ability to identify the focus of maintenance, i.e. find out where to look (using AHP), an effectiveness concept, and what to do (using Fuzzy Logic), an efficiency concept.

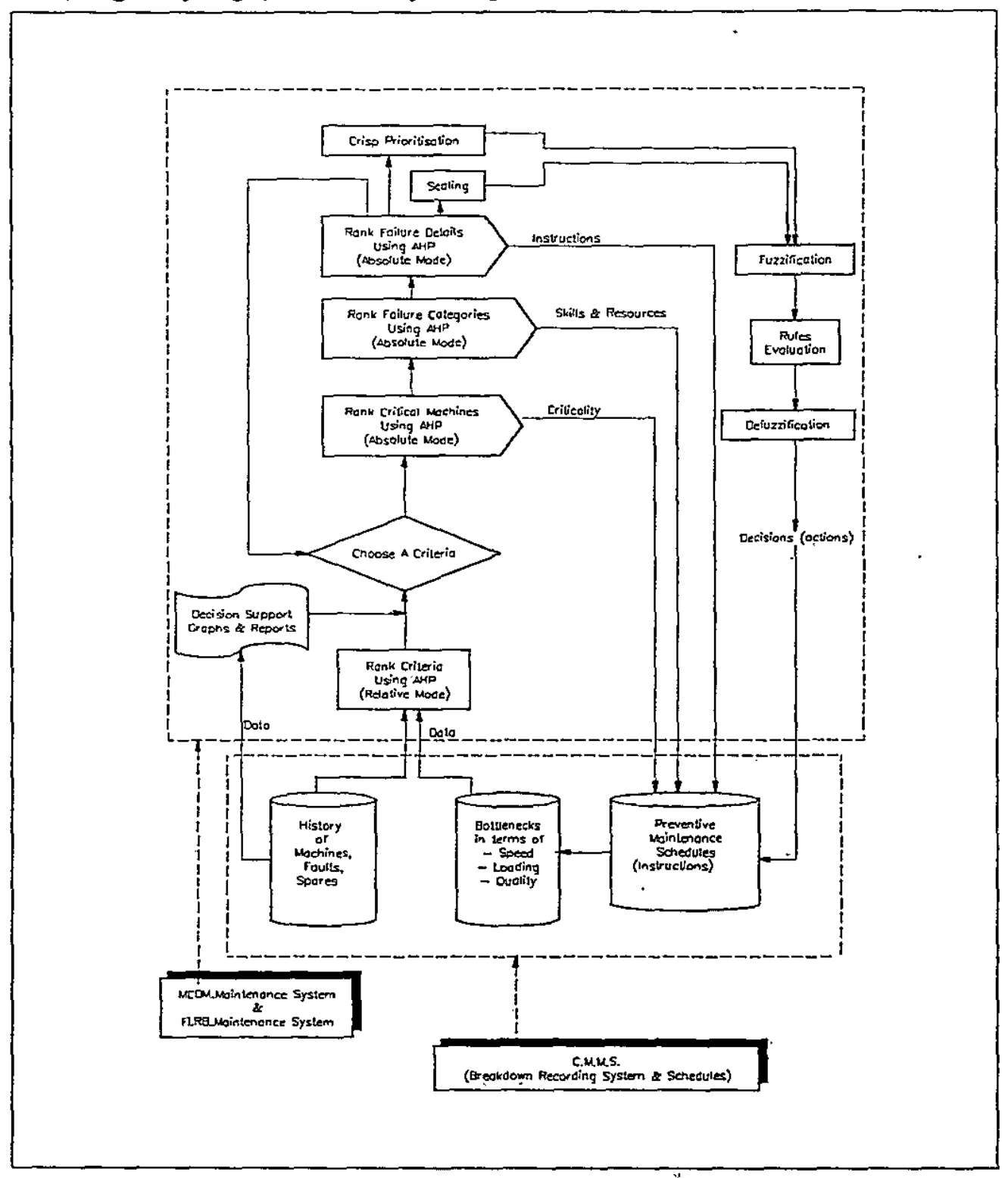

Figure (1): Flow of the System

\section{Theory Of Absolute And Relative Comparison Modes}

A core concept of this model is based on the theory of AHP, which is presented in Appendix (1). In particular, the presented model is related to the relative and absolute modes of comparison as well as the consistency ratio. In this section, an emphasis is given to the mathematical aspect of the absolute and relative modes of comparison. The AHP is a method that derives ratio scales from reciprocal comparisons. It is a method of breaking down a complex situation into its component parts, arranging these parts, or variables, into a hierarchic order, assigning numerical values to subjective judgements on the relative importance of each variable, and synthesising the judgements to determine the overall priorities of the variables. The theory of absolute and relative modes of AHP is discussed in [Saaty, 1990a]. A consistency ratio (C.R.) of 0.10 (i.e. $10 \%$ ) or less is positive evidence for informed judgement. What contributes to the consistency of a judgement are:-

(i) the homogeneity of the elements in a group, that is, not comparing a small manual machine to a big line of automated robots;

(ii) the number of elements in the group - to improve consistency the authors agree with the psychological experiments, which Saaty was able to justify mathematically [Saaty, 1990a], that an individual cannot compare simultaneously more than seven objects (plus or minus two) without becoming more and more inconsistent [Miller, 1956], as cited by Saaty; and,

(iii) the knowledge of the analyst about the problem under study. 
The proposed MCDM Maintenance model (system) includes both the absolute and the relative modes of AHP. The relative mode is used when comparing general criteria of criticality such as frequency of calls, down time, spare parts cost and bottlenecks. In addition, relative mode is used when comparing an alternative in which there is no available quantitative data, such as bottlenecks. Absolute mode is used at lower levels of the hierarchy where we have quantifiable reports on detailed faults. Notice that in absolute mode the consistency ratio C.R is always equal to zero, i.e. complete consistency, since we have the exact value of $w_{i} / w_{j}$ in the comparison matrix. The two principles of (i) homogeneity, and (ii) number of alternatives, were maintained through performing a pareto analysis of each alternative with respect to the different criteria (Top 7 reports with respect to downtime, number of calls, and spare parts cost). Here, it is assumed that the decision maker agrees with the TPM concept of eliminating the faults starting with the worst machines, and hence assures achieving maximum gains. In the lower level of fault details, the maximum number of 7 categories and their details were maintained, and found practically sufficient to represent failure modes with considerable accuracy.

\section{The M.C.D.M. System (Model) Structure}

The presented system works in three stages. The first stage involves extracting decision support reports to assess decision makers' evaluation of different criteria. Once criteria are identified and investigated, the second stage is to prioritise different criteria by performing a multiple criteria evaluation method. The evaluation is carried out in a structured approach using the Analytic Hierarchy Process (AHP) methodology, and it is intended that this stage be conducted in a group decision making environment. Based on different criteria, machines are ranked according to criticality in the relative mode, followed by an in-depth focused analysis of failure details in a graphical and a hierarchical format and in the absolute mode. Figure (2) shows the screen of the main hierarchy. The model is structured as a hierarchy with a focus (objective) of determining criticality. Hence, critical machines and their most critical fault details are obtained based on different criteria. The consideration of different criteria assures an effective overall picture, and a near optimum solution.

The first level is criteria evaluation, where the decision maker prioritises his/her preferences of different criteria such as frequency of calls, downtime, spare parts cost, and bottleneck machines. It should be emphasised here, that the criteria presented in this model (system) are not comprehensive, and can be modified to suit different companies. However, the model serves as a framework of a methodology to determine criticality. In addition, the presented criteria were chosen since they combine both quantitative data and qualitative judgements.

Once criteria are prioritised through using the AHP algorithm in relative comparison mode and hence knowing his/her consistency, the decision maker can move to the second level of the hierarchy to find the most critical machines (Pareto analysis) based on any of the above mentioned criteria. The machines are then ranked according to their weights. Weights are obtained through running an AHP algorithm in an absolute mode, and hence a consistency ratio of value zero is assured. This means an ideal consistent judgement. Details on any of these critical machines in terms of failure events, their duration, and spares replacements, can be easily extracted as decision support reports. Once the most critical machine (based on any criteria) is identified, the decision maker can move to the third level where the distribution of failure categories are given. Failure categories are grouped into general ones such as mechanical, electrical, hydraulic, pneumatic, and coolant type of failures. From now on the ranking is always performed using the AHP algorithm in the absolute mode of comparisons. At this stage the decision maker can have a broad idea about the distribution of different types of failures. This helps to identify strategic areas where different maintenance trades and/or skills are needed.

The fourth level of the hierarchy is concerned with specific faults related to each of the fault categories mentioned in the previous paragraph. These faults are related to major sections of the fault categories. For example, under the category of mechanical faults, faults related to sections such as head stock, fixture, slide, axis drive, turret, conveyor,..etc are given and ranked according to criterion specified in level 1 of the hierarchy. The final two levels (level 5 , and 6 ) are concerned with detailed failure components of the major sections in level 4. For example, the head stock section (level 4) of the mechanical category (level 3) is composed of components such as bearings, clamping, gibbs, screws, index, and motor. As shown, using this hierarchical decomposition the decision maker is able to focus on specific detailed faults at the lowest level of the hierarchy, but starting from a general, fuzzy, and non-quantifiable set of criteria at upper levels of the hierarchy. This attention to detail in a structured manner is the basic philosophy behind the concept of TPM in an appropriate, systematic, and adaptive approach. Once taking actions towards minimising these faults, or losses, a maximisation of overall effectiveness will materialise. 


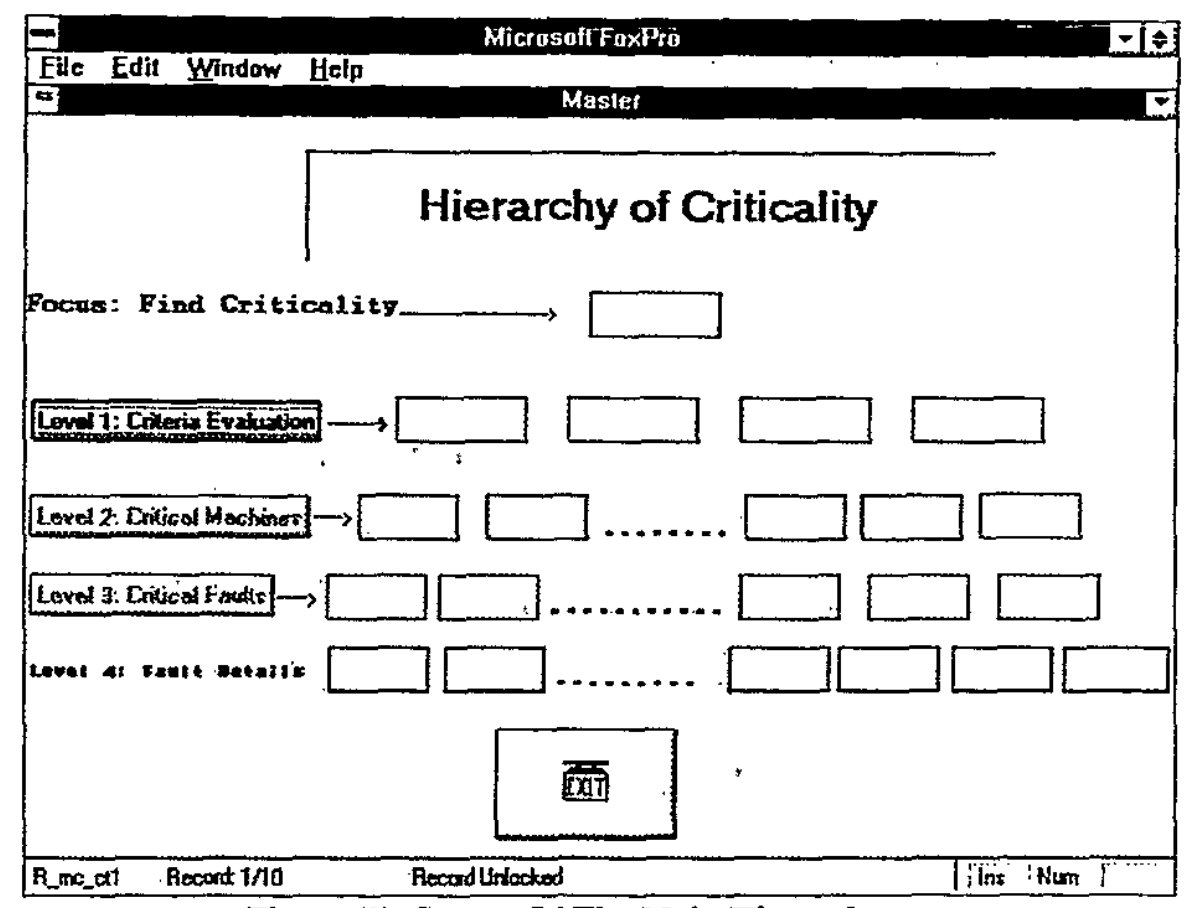

Figure (2): Screen Of The Main Hierarchy.

Criteria Analysis

It has been noticed that the maintenance problem can be formulated in a multiple criteria decision making approach. These multiple criteria are due to the fact of existing multiple groups involved in maintenance, or in other words, maintenance as a function, is not isolated, but interact with other functions such as production, finance, and quality. These functions often operate according to multiple objectives, and are often of a conflicting nature. As an example, critical machines can be looked at; from the point of view of maintenance personnel, in terms of the most frequent maintenance calls. This means that the machine with highest frequency of events is considered the most critical, since maintenance resources are allocated to each of these events. On the other hand, the production personnel are more concerned with machines that break down for longer times, and hence downtime is one of their major criteria as well as being a bottle neck machine due to its capacity, quality, or number off. Finally, people in charge of finance will base their criticality criteria on machines that consume more spare parts cost. Hence, it is obvious that decision making in maintenance should be considered from a multiple criteria perspective, and these criteria need to be prioritised, and priorities can be changed over time. The following criteria were chosen because as mentioned before, they have been found to be of major concern to maintenance, production, finance, and quality personnel. In addition, they combine both quantitative and qualitative preferences, and hence shows the power of this approach. The developed system (model) consists of four criteria:

i. Frequency. Number of maintenance calls (break down events).

ii. Down time. Downtime in hours.

iii. Spare parts cost. Cost due to ordering for replacement of spare parts.

iv, Bottle necks and/or quality. Judgmental preference based on loading, number off, cycle time, ...etc.

The first step in studying the criticality is to find out how critical are the critical machines. In other words, to find out how much of the total trouble is caused by the critical machines. This total view will help in visualising the perceived benefits that will result from minimising the effect of these critical machines. When applying the MCDM Maintenance Model to existing Computerised Maintenance Management System CMMS, it is easy to get quantitative data relating to frequency and duration of maintenance breakdowns as well as spare parts costs. However, since most CMMS are not related to production systems such as MRP systems, it was found that issues related to production bottlenecks to be of a qualitative nature. Other criteria can include issues such as safety, and machine health in terms of condition monitoring. These criteria can be investigated by pressing the relevant push button in the system to extract a decision support report, as shown in Figure (3). An important concept in the system is that data produced as an output of any report is saved in a new database for further analysis in lower levels of the hierarchy. 


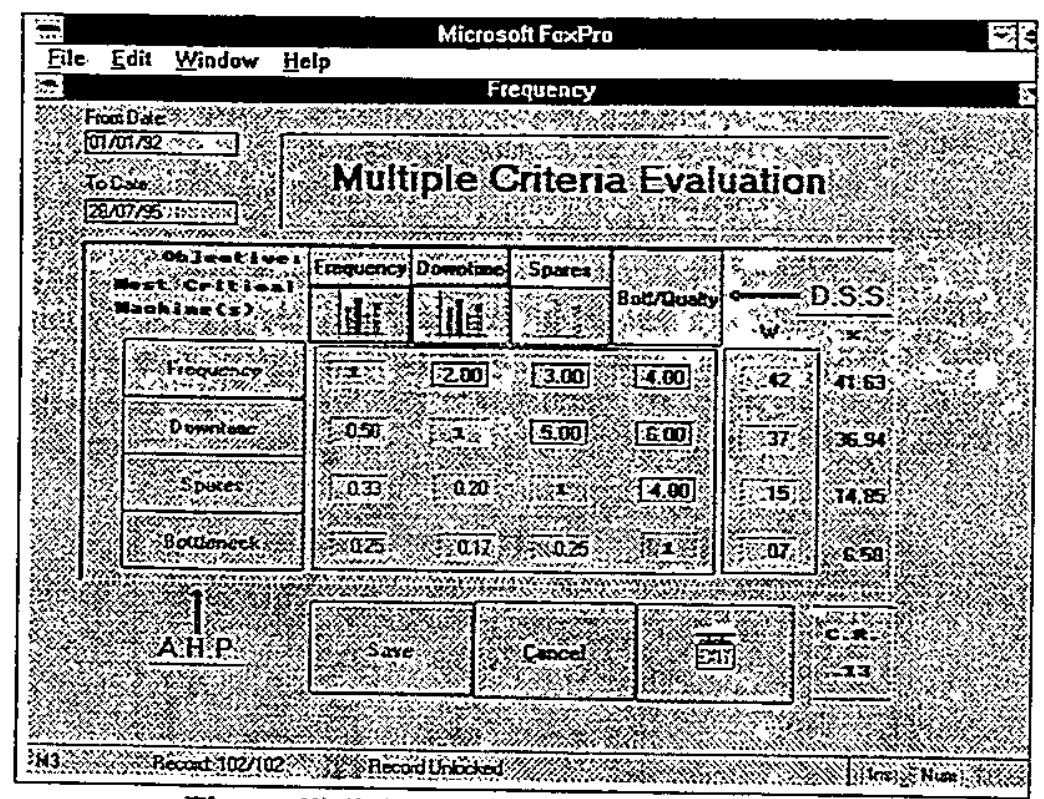

Figure (3): Multiple Criteria Evaluation.

\section{Frequency Criteria}

Frequency of calls is usually the criteria that concerns the maintenance supervisor since an initiated work order for a machine will mean that a maintenance engineer, or a crew, will have to respond and investigate reasons for the call. Frequency figures are given in either a Pareto report format or in a graph format as shown in Figure (4). Notice that, in this particular factory the worst ten machines as shown in Figure (4) were found to comprise about $35 \%$ of the total number of faults, given that there are more than 700 machines. Thus, if adequate preventive maintenance instructions are implemented, improvement of the amount of this percentage can be attained.

\section{Figure (4): Pareto of Top Ten Worst Machines Based on The Criteria of Frequency of Calls}
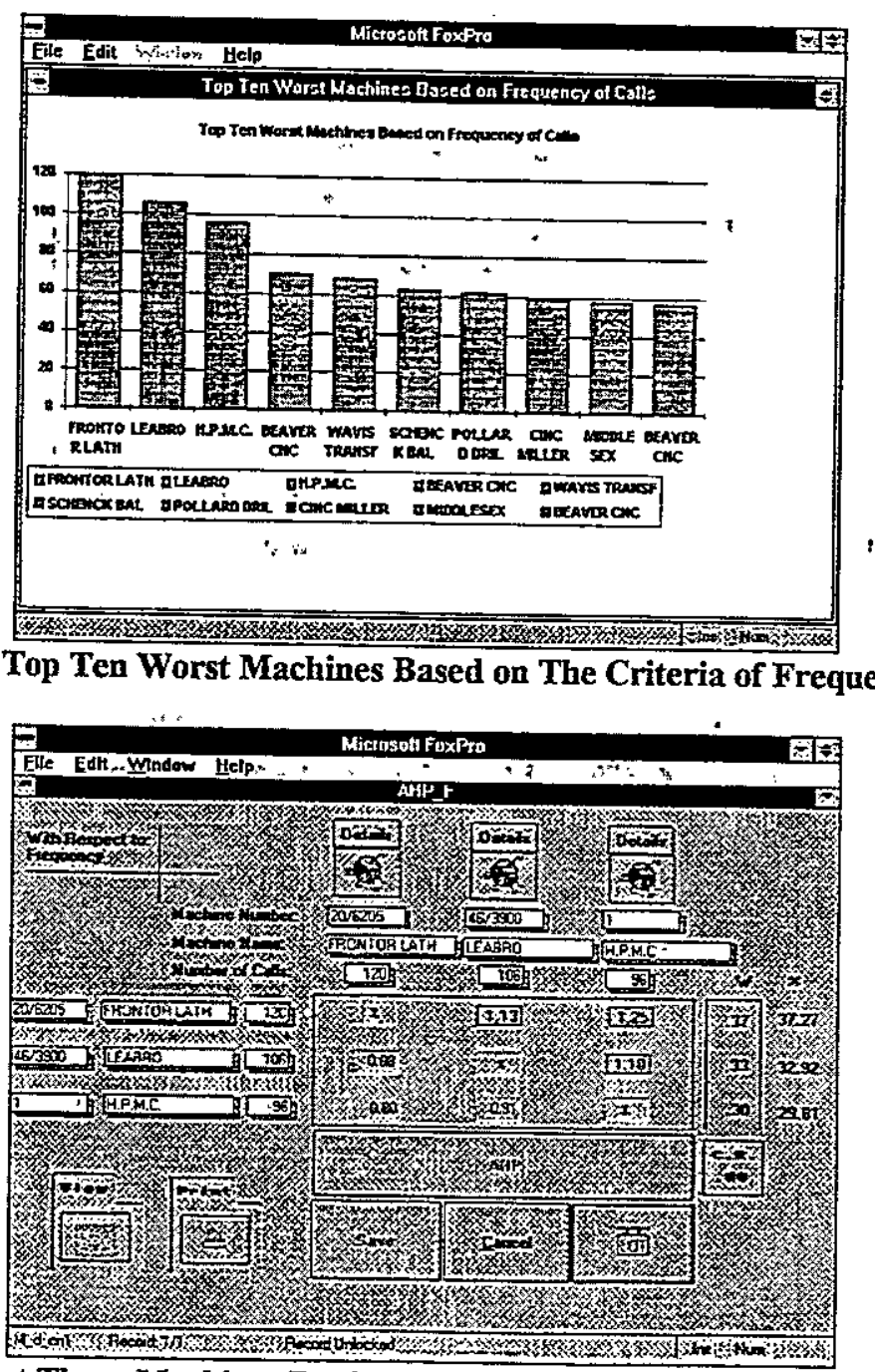

Figure (5): Top Worst Three Machines Ranked According to Frequency of Calls Criteria 
The constraint on the size of the screen is the reason for choosing only three machines for further analysis. All machines in the pareto can be further analysed if the screen size permits. In this analysis the AHP is performed in an absolute mode since their exact number of calls are known from the available data. This is shown in Figure (5). Notice that in this screen the consistency ratio is zero i.e. perfectly consistent, since the exact value of frequencies are known. Further analysis on a particular machine of the above three machines, and based on the criteria of frequency of calls, can be performed by either extracting a detailed report as shown in Figure (6), or by pressing on the graph push button where a graph of the distribution of failure categories is given as shown in Figure (7). The report shown in Fig. (6) illustrates maintenance events (jobs) carried out for a particular machine. As shown, any horizontal line gives data on the reported fault from production, and the actual fault diagnosed by maintenance personnel. It also shows the downtime duration and the date of the event. In this report data is arranged into groups of similar faults as diagnosed by maintenance.

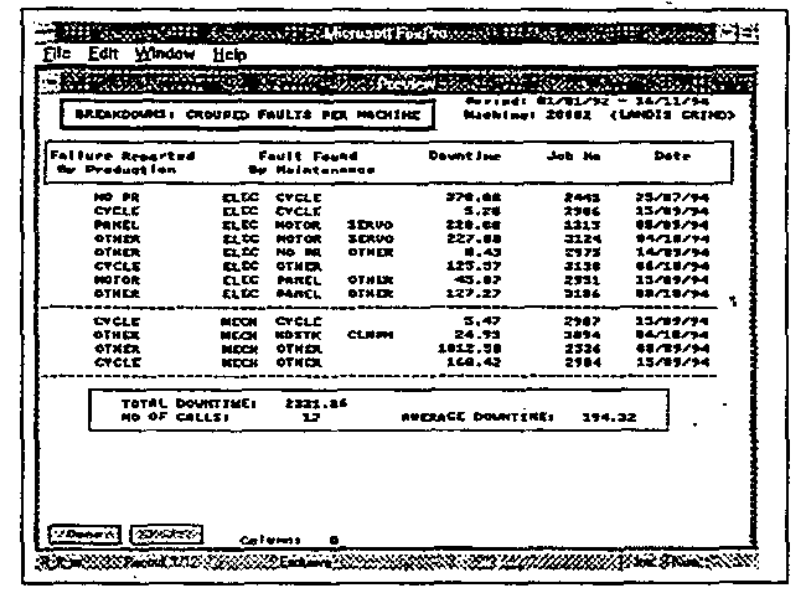

Figure (6): Report of Failure Categories Of A Specific Machine Based on Frequency Criteria

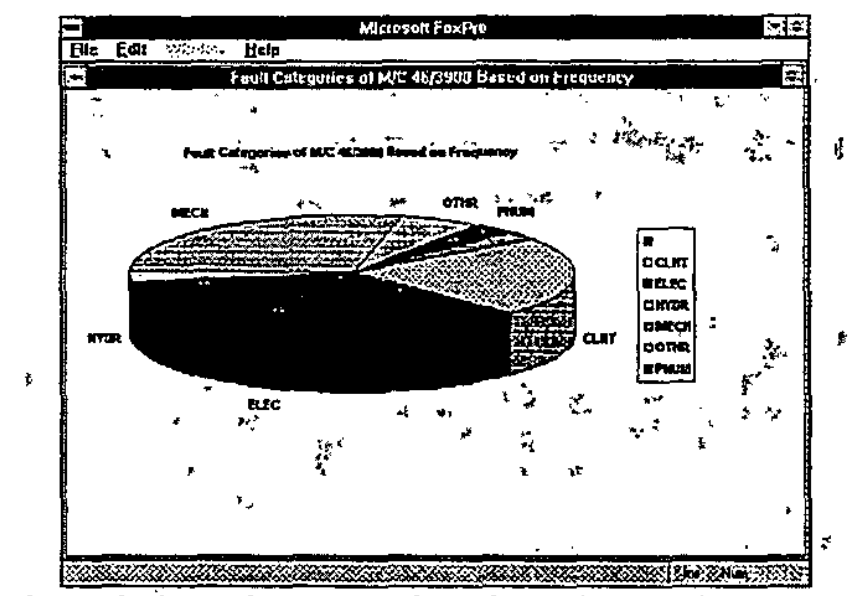

Figure (7): Distribution of Failure Categories Of A Specific Machine Based on Frequency Criteria

The decision maker can then move to more detailed levels of fault analysis for the chosen machine and based on a specific criterion (frequency in this case). This is shown in Figure (8). The AHP algorithm is performed on the failure categories. The same concept of failure details is carried out in a multi-leveled approach.

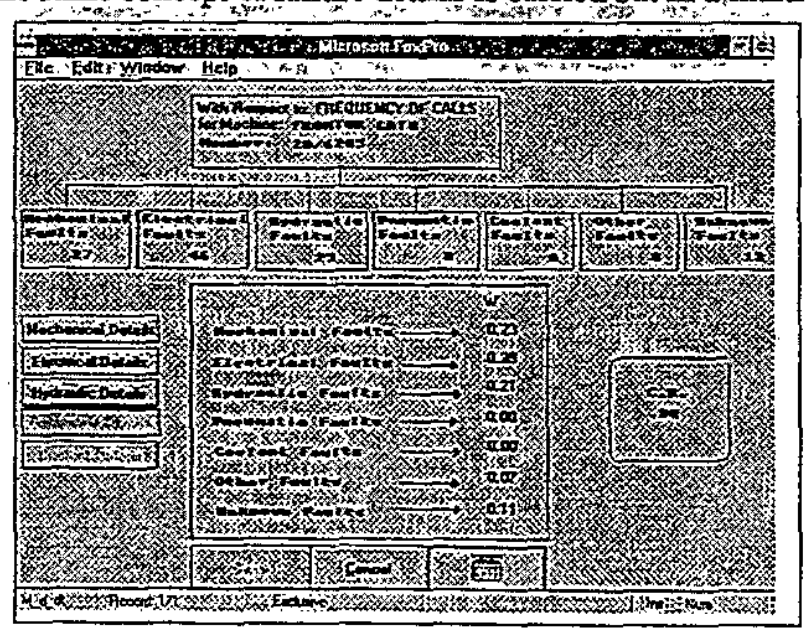




\section{Downtime Criteria}

The downtime hierarchy is the measure of availability of equipment. It often concerns the production manager since it means less available capacity for production. Downtime figures are given in either a graph format or in a Pareto report as shown in Figure (9).

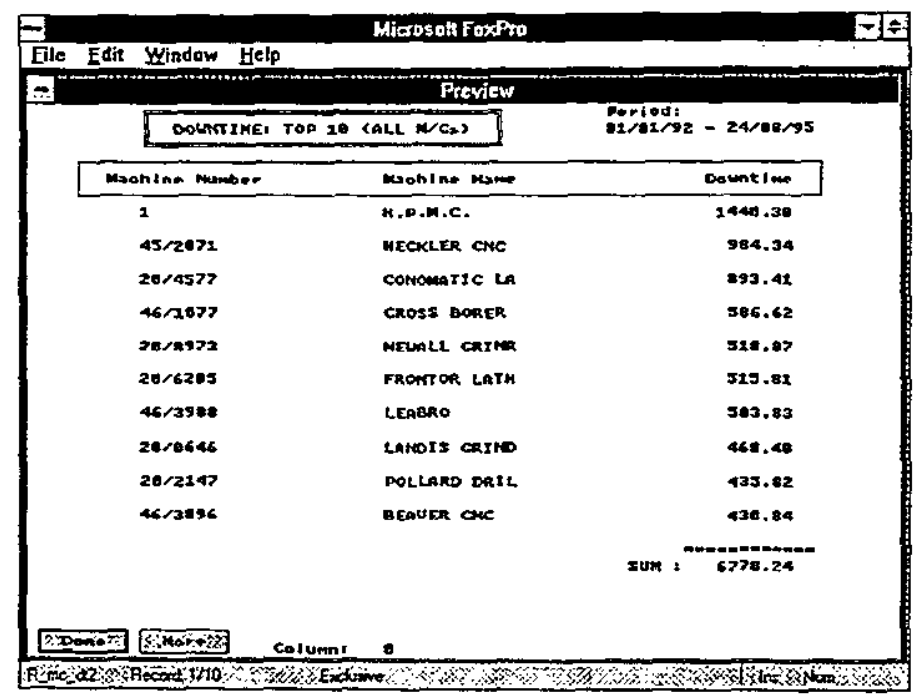

Figure (9): Report of Top Ten Worst Machines Based on The Criteria of Downtime

\section{Spare Parts Cost Criteria}

Although much of research and practice related to the maintenance function has ranged from failure analysis in terms of condition monitoring and diagnosis to strategic aspects such as TPM and information systems, very little effort has been exerted to solve the root cause of what might be a catastrophic situation facing maintenance, and that is lack of spares [Neyret, 1994]. One must remember that when a vital spare part, needed to repair critical equipment, is missing, the maintenance engineer - no matter how skilful he/she may be, or how effective his/her organisation is - will be helpless until he/she obtains that spare part, which may be several months later. Thus, it is an important criteria to consider, especially if there are available data. Details of spare parts consumption for a specific machine are shown in Figure (10). Notice that knowing the number and value of spares used, one can estimate the stock level needed to keep in hand to avoid equipment stoppages due to missing spare parts.

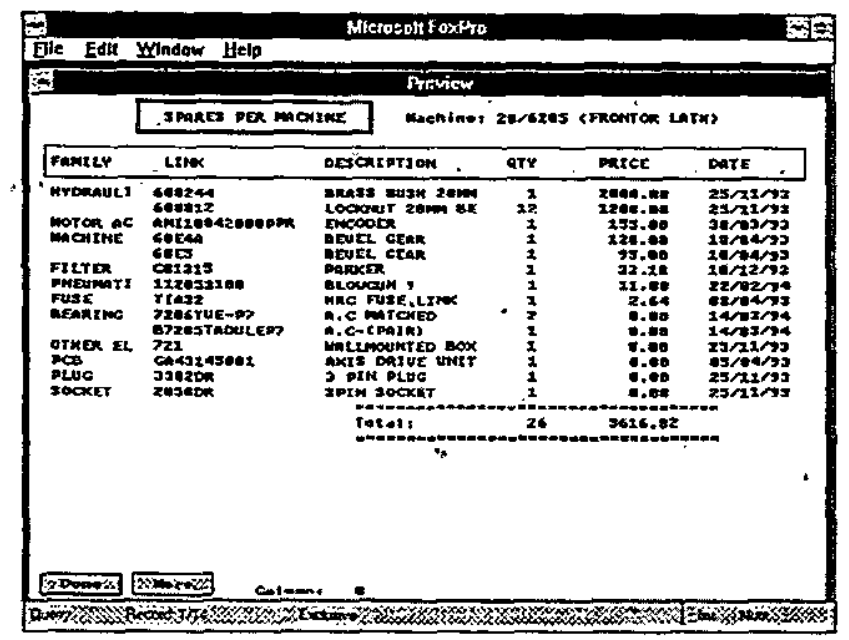

Figure (10): Spare Parts Replaced In A Specific Machine

\section{Bottle Necks Criteria}

A good understanding of the role of a bottleneck machine helps in correctly assessing the cost of downtime resulting from failures of this machine or related ones. Bottleneck criteria can be assessed based on different sub-criteria such as number off, capacity constraint (throughput), quality (scrap rate), or the cycle time of the preceding machine (to minimise working process in a JIT environment). Since it is difficult to quantify all these factors and their combined effect, the decision maker is given the choice to select machines from the asset register. The decision maker can then compare the criticality of the chosen machines in terms of being bottlenecks using AHP in the relative mode. Notice that the decision maker will base his/her judgements on his/her qualitative, and subjective preferences in the relative mode, and hence consistency ratio (CR) is not necessarily equal to zero. 


\section{Fuzzy Logic Rule - Based Maintenance System (F.L.R.B. Maintenance )}

In the model (system) developed the crisp inputs are frequency of calls and downtime of a specific component. Additional inputs such as spare parts cost, production losses, and so on can be added using the same algorithm. The values of these crisp inputs are extracted through running the MCDM_Maintenance model for any component as described in a previous section. The crisp output is which specific action to follow based on given IF-THEN rules and a cost function. The link between the two models MCDM_Maintenance (based on AHP), and the FLRB_Maintenance (based on Fuzzy Logic) can be illustrated in figure (11). Two important types of outputs from the MCDM system are required as inputs to the FLRB system. The first type of output is crisp prioritised values for criteria such as downtime, and frequency of calls. The second type of output is a sense of scale, where one can determine what to be considered small, medium, and high for downtime and frequency of calls. This is an important requirement for obtaining the scale of the membership function in the fuzzification process, as will be shown below.

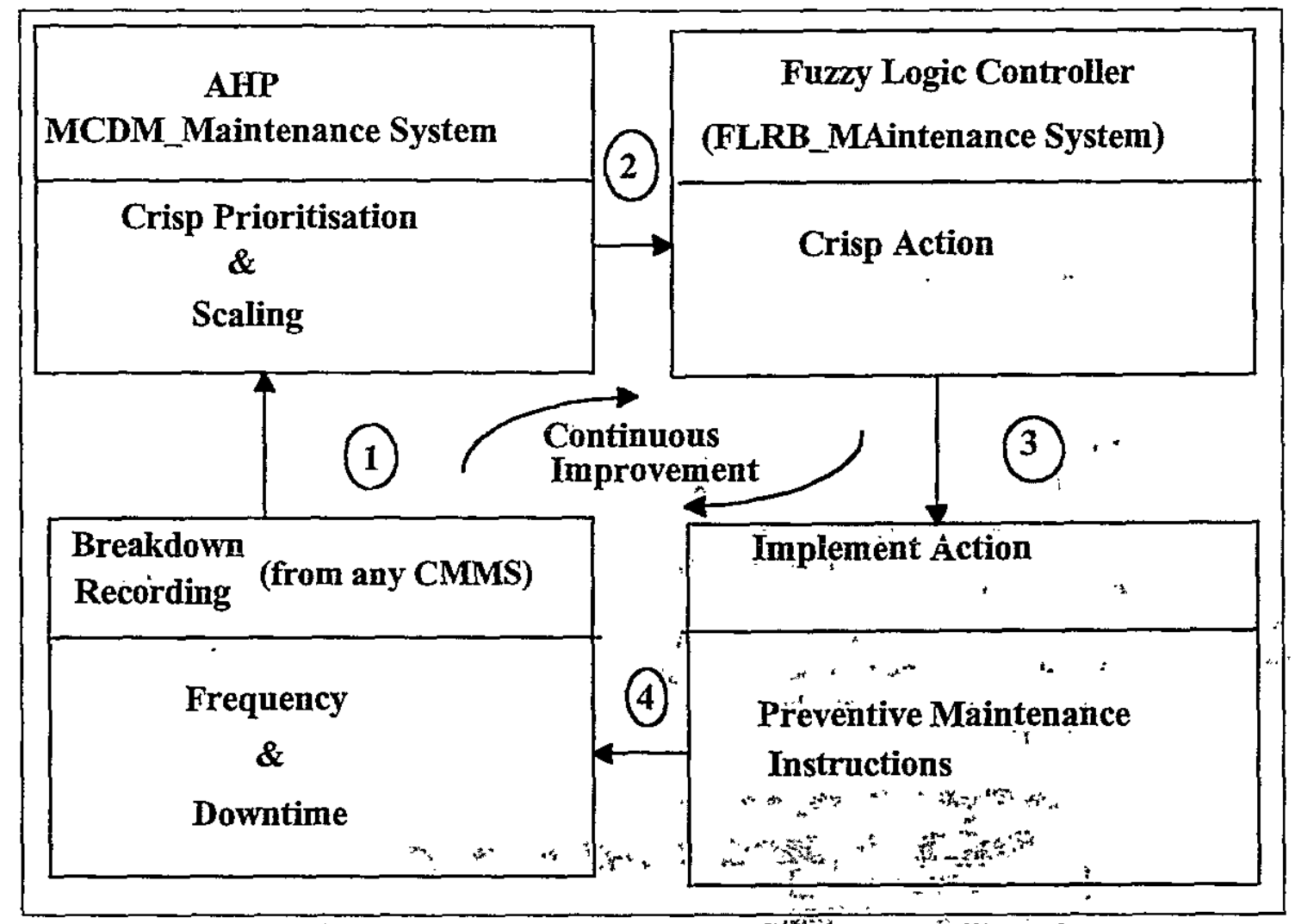

Fig. (11): The Link Between The MCDM.System and the.FLRB System

An example of different maintenance actions is given below. The proposed.FLRB model can be summarised as shown in Figure (12). The three steps of the furzy controller; as shown in Fig. (12); are:

i. Fuzzification; ii. Rule evaluation; and iii.Defuzzification."

Each of these steps is described below.

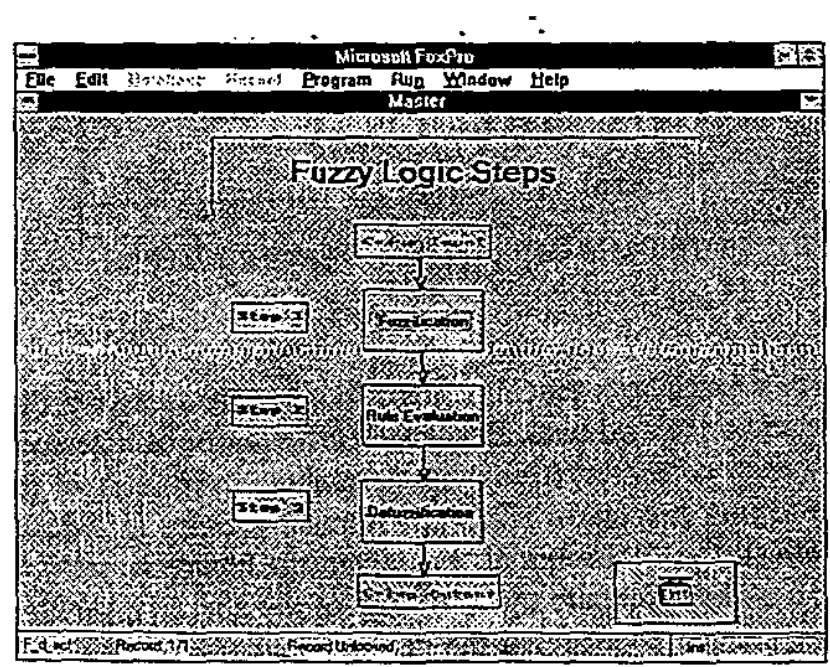




\section{First Step: Fuzzification}

The first step in the fuzzy controller is the fuzzification process.

The membership function, universe of discourse $U$, is the classifications that are considered in the problem. It is assumed that both frequency and downtime can be classified into 'High', Medium' and 'Low'. However, each type of input has its specific scale. The decision maker should be able to specify a different scale for each case according to his/her preferences, which are obtained from experience after nunning the MCDM_Maintenance system. In Fig. (13), the membership function for the frequency (number of calls set) is illustrated. A trapezoidal function is assumed for simplicity of coding. However, the model can be adapted to deal with non-linear functions.

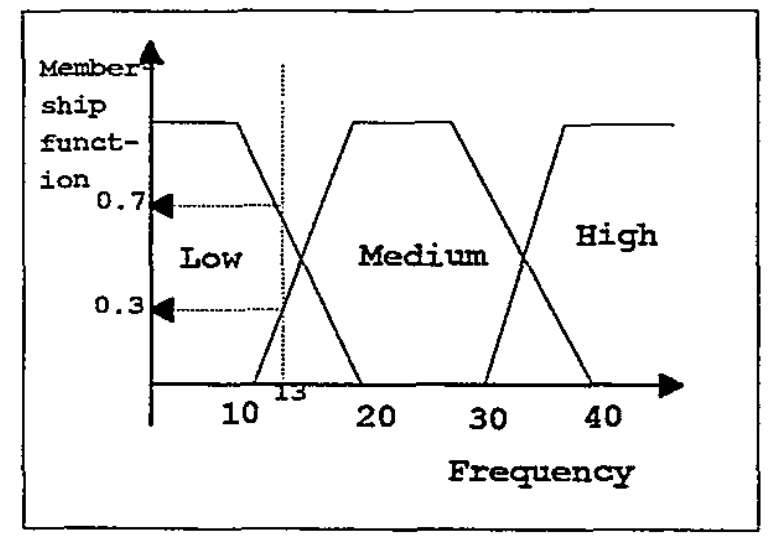

Fig. (13): Membership of fuzzification process

The algorithm of fuzzification is implemented in the FLRB_Maintenance system developed as shown in Fig. (14). Let the fizzy inputs $\mu$ for frequency be $\mu_{f l}, \mu_{f m}, \mu_{f h}$, and that for downtime be $\mu_{d l}, \mu_{d m}, \mu_{d h}$ for the low, medium, and high values respectively. Notice that given the scales of frequency and downtime, and given crisp values of 13 for frequency, and 360 hours for downtime, one can obtain fuccy values of $0.7,0.3$, 0.0 for $\mu_{f l}, \mu_{f m}, \mu_{f h}$ respectively, and $0.0,0.4,0.6$ for $\mu_{d l}, \mu_{d m}, \mu_{d h}$ respectively, as shown in Fig. (14). Notice that the values of membership $\mu$ are in the interval of $[0,1]$.

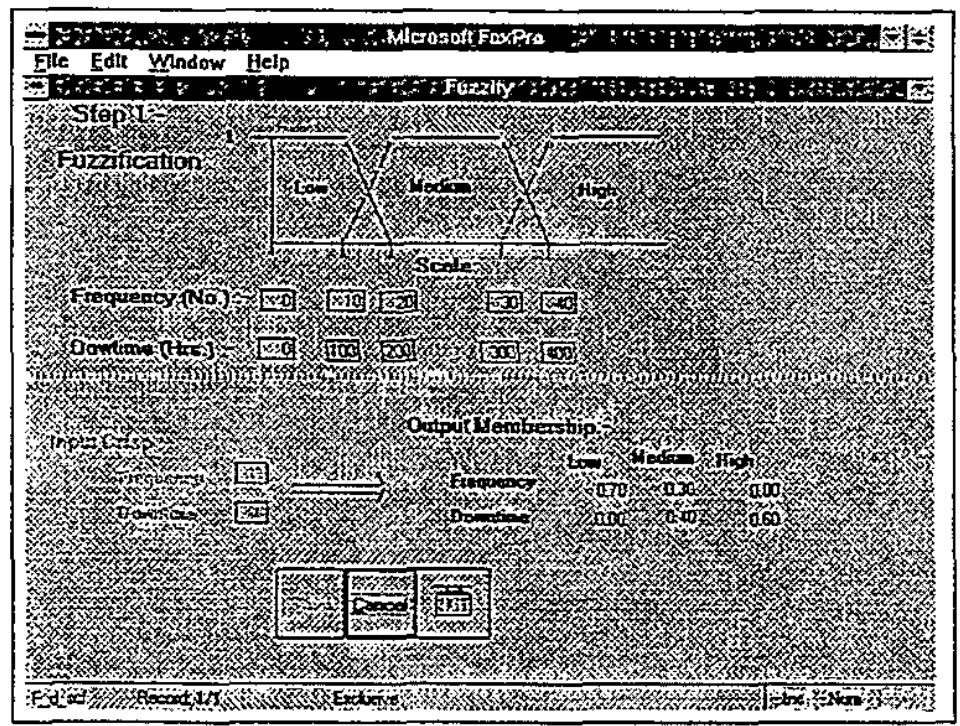

Fig. (14): Fuzzification of Frequency, and Downtime (Screen of the System).

\section{Second Step: Rule Evaluation}

Referring to Fig. (12), the rule evaluation step can also be explained as an input-output system. In this step, inputs are expert rules, and fuzzy inputs obtained from the first step (i.e. values of $\mu$ ), while outputs are furzy values of maintenance actions to be carried out. Given two variables of frequency and downtime with each having three subsets of Low, Medium, and High, then one needs at least nine (3x3) rules to describe the model (system). These rules are in the form of IF..THEN..statements. Based on experience, and actions 
provided by [Henry, 1993], and [Pintelon, and Gelders, 1992] examples of maintenance prescriptions are as follows:-

i. Operate To Failure (OTF), ii. Fixed Time Maintenance (FTM), iii. Skill Levels Upgrade (SLU), iv. Condition Base Monitoring (CBM), and v. Design Out Maintenance (DOM). Notice that the above actions are not comprehensive, and can change according to the environment of each company. The objective is to present a framework for developing rules for the fuzzy controller. A summary of the application of each action, based on the values of Frequency (Fr) and Downtime (Dt), is given in Table ( 1 ).

\begin{tabular}{|l|l|c|}
\hline \multicolumn{1}{|c|}{ Name } & \multicolumn{1}{|c|}{ Application } & Cost Code Function \\
\hline OTF: Operate To Failure & $-\mathrm{Dt}=$ Low,$\quad$ Fr $=$ Low & $(10)$ \\
\hline FTM: Fixed Time Maintenance & $-\mathrm{Dt}=$ Medium, Fr $=$ Medium & $(20)$ \\
\hline CBM: Condition Base Monitoring & $-\mathrm{Dt}=$ High, $\quad \mathrm{Fr}=$ Low & $(30)$ \\
\hline SLU: Skill Levels Upgrade & $-\mathrm{Dt}=$ Low, $\quad \mathrm{Fr}=$ High & $(40)$ \\
\hline DOM: Design Out Maintenance & $-\mathrm{Dt}=$ High,$\quad \mathrm{Fr}=$ High & $(50)$ \\
\hline
\end{tabular}

Table (1): Summary of Maintenance Actions \& Their Application

An example of a rule can be "IF both downtime and frequency are low, THEN do nothing". In other words the component, or machine, can operate till it fails since it seldom fails. This rule can be written as follows:

"IF Frequency is LOW and Downtime is LOW THEN O.T.F." （Rule 1).

The summary of rules are presented in Table (2). Notice that if the action is implemented successfully, it is expected that the next state should be a movement towards the north-west direction. In other words, the objective is to transfer the condition of a particular machine (or component) from high to medium and from medium to low for both frequencies and downtime.

\begin{tabular}{|l|c|c|c|}
\cline { 2 - 4 } \multicolumn{1}{c|}{} & Dt (Low) & Dt (Medium) & Dt (High) \\
\hline Fr (Low) & OTF & FTM & CBM \\
\hline Fr (Medium) & FTM & FTM & FTM \\
\hline Fr (High) & SLU & FTM & DOM \\
\hline
\end{tabular}

Table (2): Summary of Rules for Maintenance Actions

In the FLRB_Maintenance system (model) rules are constructed as shown in Fig. (15) at the upper left box of the screen.

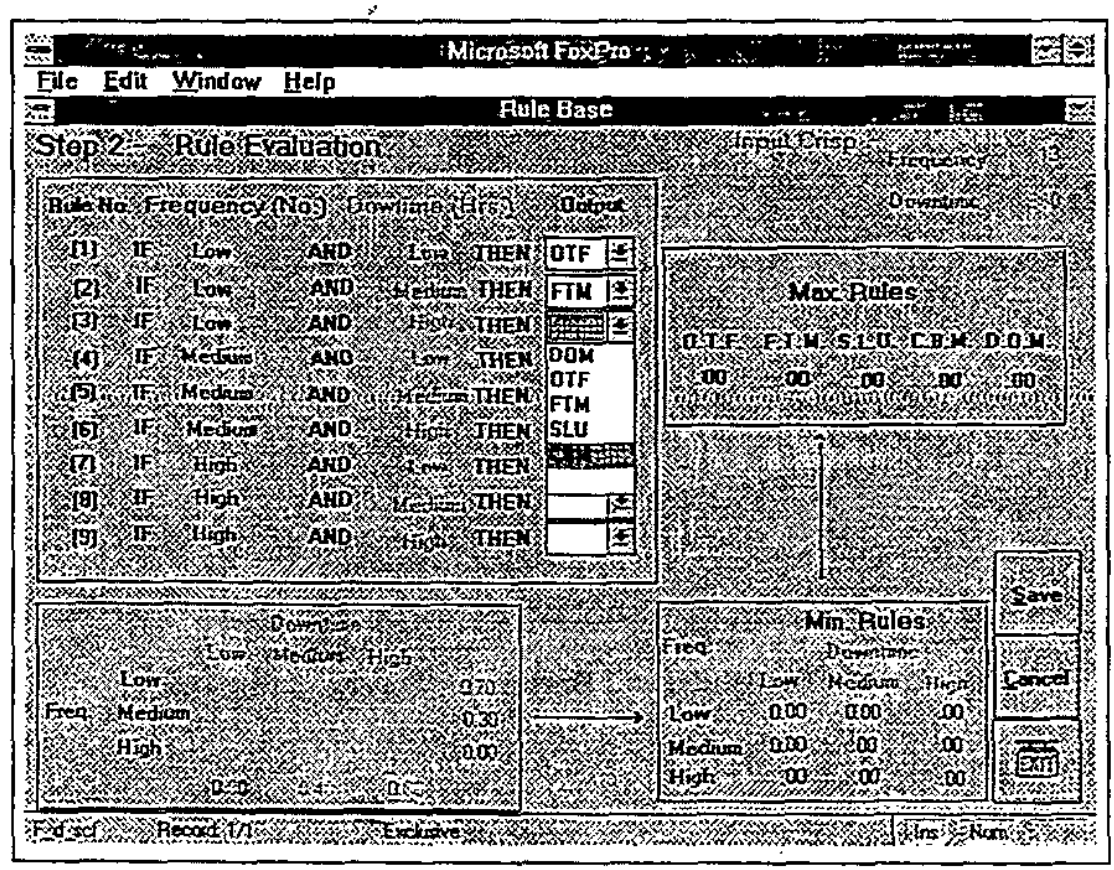

Fig (15): Rules Construction in the System.

Once rules are constructed, and given the values of the fuzzy inputs for $\left(\mu_{f l}, \mu_{f m}, \mu_{f h}, \mu_{d l}, \mu_{d m}, \mu_{d h}\right)$ one can apply the min. \& max. (AND \& OR Zadeh) inference computations. The combination of rules, and the fuzzy input values is shown in Fig. (16). 


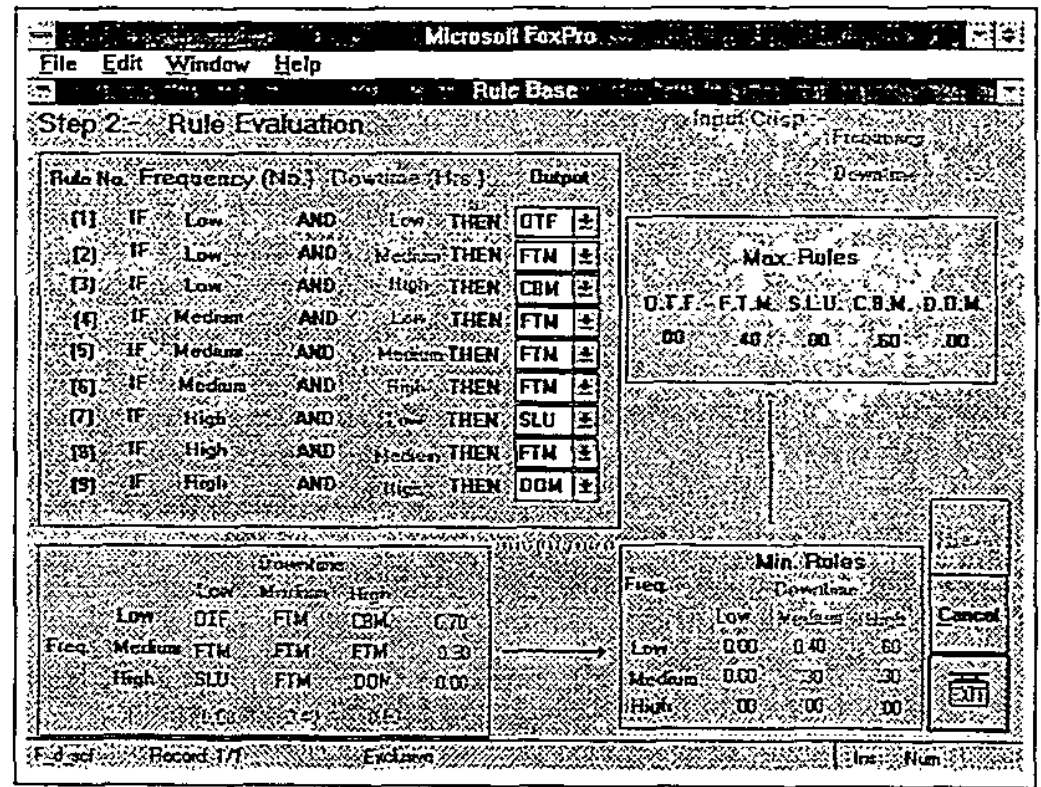

Fig. (16): A Screen of Rules Evaluation.

\section{Third Step: Defuzzification}

The final step in the fuzzy controller is the defuzzification process. This process is based on the idea of deriving a crisp value for a fuzzy function. These items are arranged according to the degree of difficulty and cost of implementation, starting from the simplest to the more difficult ones. The defuzzification can be performed by deriving the centre of gravity of the area under the curve of the function. Given the cost function of each maintenance action, one can arrange the maintenance actions, the fuzzy output, and the cost scale function as shown in Fig (17).

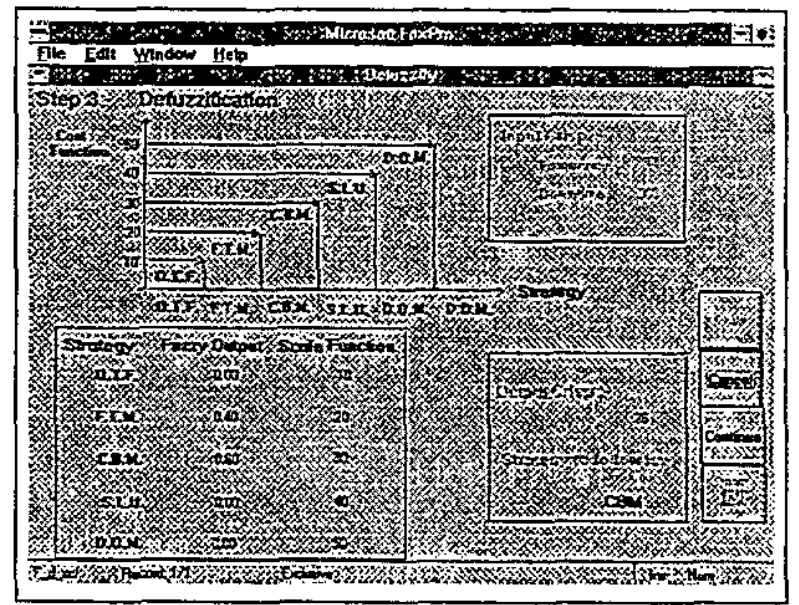

Fig (17): A Screen of Defuzzification.

Since the value of (26) is between FTM and CBM on the function scale, then the recommended action to follow is (40\% FTM, and 60\% CBM). Hence, for a particular machine (or fault), given two crisp inputs of frequency and downtime of the values (13 events, and 360 hours), one should allocate $60 \%$ of the time, or money on monitoring the condition of this machine, and $40 \%$ on a fixed time maintenance (FTM) i.e. performing basic instructions on an equal interval of time. If according to these actions the number of failures and their duration decrease, which means that the state of the machine has moved towards the north-west direction in the rules grid as shown in Table (2), then the output of the system will be expected to be (OTF) as an action to follow, hence, (FTM) \& (CBM) should be terminated as the machine is being over-maintained. The feedback mechanism offered by the rules grid of Fuzzy Logic, as shown in Table (2), in addition to the feedback already offered in AfIP in the form of consistency ratio, provides a near optimum performance.

This model coincides with most of the specifications of an intelligent decision support system properties as identified by a recent work of [Kobbacy et al., 1995]. In addition, it fulfils the framework outlined by [Vanneste, And Wassenhove, 1995], which concerns a systematic procedure seeking effectiveness. 


\section{Advantages Of The Model (System)}

Advantages of the proposed model can be described as a feedback mechanism which can be illustrated by the following example: An aeroplane, no matter how much power, or fuselage design or speed will not be able to operate unless the pilot has in his/her panel indicators that give him/her a feedback on the performance, location, altitude, pressure, and others. The same is applied to manufacturing as well, a guided and focused approach with a feedback mechanism is needed. The proposed model serves as an approach to monitor performance, and to provide a focused feedback. The system offers a contribution towards integrating preventive and corrective modes of maintenance, since it suggests focused actions that ought to be carried out as preventive instructions and based on a real-time response to corrective modes. The system offers an adaptive and dynamic framework, and hence production and maintenance are integrated in a 'real time' environment. The system is also flexible in working on any other criteria whether it is of a quantitative or qualitative nature. Hence, it can be integrated with different kinds of maintenance systems for example, condition monitoring where criteria such as vibration, pressure, temperature, speed and others can be considered and priorities of machines can vary alternatively. As mentioned earlier the criteria described are of major concern to different people. Hence, the system can be considered as a structured environment for group decision making. As the group move downward along the hierarchy, the system focuses on details of faults. This attention to detail is the core of the philosophy behind the TPM concept.

In short, the model described above promotes strategic maintenance decisions. It seeks effectiveness through prioritising machines' criticality, and focusing on specific components that will maximise gains based on different criteria. In the next paper this model is extended through the development of fuzzy logic nule-base controller that seeks an efficient approach to specify the most appropriate maintenance action to follow based on different rules.

\section{References}

- Cho, D.I., and Parlar, M. (1991) "A Survey Of Maintenance Models For Multi-Unit Systems", European Journal Of Operational Research, Vol. 51.

- Dyer, Robert F., And Forman, Ernest H. (1991) "An Analytic Approach To Marketing Decisions", Prentice-Hall, Inc.

- Henry, T. A. (1991) "Availability Centred Maintenance", The Plant Engineer, 37/4, July/August.

- Hintz, G.W., and Zimmermann, H.J., (1989) "A Method To Control Flexible Manufacturing Systems", European Journal Of Operational Research, Volume 41, No.3

- Kobbacy, K. A. H., N.C. Poudlove, and M.A. Harper, (1995) "Towards An Intelligent Maintenance Optimisation System", J. Of The O. R. Society, 46/7, July, pp 831-853.

- Laakso, Kari, Sheppo Hanninen, And Kaisa Simola, (1995) "Experience-Based Reliability Centred Maintenance", Maintenance Journal, Vol. 10, No. 1, Jan/Feb., pp 3-7.

- McKinnon, Sharon (1992) "The Information Mosaic", ppl62-164, McGraw-Hill.

- Miller, G.A. (1956) "The Magical Number Seven Plus Or Minus Two: Some Limits On Our Capacity Of Processing Information", Psychological Review 63 pp 81-97.

- Neyret, G (1994) "The Supply Of Spare Parts: A Growing Problem For The Maintenance Engineer", Maintenance Journal.

- Pintelon, L.M. and L.F. Gelders (1992) "Maintenance Management Decision Making", European Journal Of Operational Research, Vol. 58.

- Saaty, Thomas L. (1990a) "Multicriteria Decision Making - The Analytic Hierarchy Process - Planning,, Priority Setting, Resource Allocation", RWS Publications, Pittsburgh, USA.

- Saaty, Thomas L. (1990b) "Decision Making For Leaders: The Analytic Hierarchy Process For Decisions In A Complex World", 2 nd edition, RWS Publications, Pittsburgh, USA.

- Saaty, Thomas L., and Vargas, Luis (1991) "The Logic of Priorities Applications of The Analytic Hierarchy Process In Business, Energy, Health, \& Transportation", 2 nd edition, RWS Publications, Pittsburgh, USA.

- Vanneste, S.G., and Van Wassenhove, L. (1995) "An Integrated And Structured Approach To Improve Maintenance", European Journal Of Operational Research, 82/2.

Appendix (1): Theory of the Analytic Hierarchy Process (AHP).

The theory of AHP is based on the concept of having $n$ alternatives and their relative pair-wise comparison $a_{i j}$ is an approximation to the ratio of $w_{i} / w_{j}$ which is the weight of alternative $i$ to alternative $j$. The decision maker does not know in advance the value $w_{i} / w_{j}$. The hierarchy normally cônisists of a top nóde :the goal, then the second layer is the criteria level, and finally the third layer is the alternatives level. The basis of the AHP is the completion of an $i x j$ matrix at each level of the decision hierarchy. This matrix $A$ is of the form $a_{i i}=1 / a_{i i} a_{i i}>0$ : i.e. $A$ is a positive. recibrocal matrix. The basic theorv is based on the fact 
that $a_{i j}$ is an approximation to the relative weights $\left(w_{i} / w_{j}\right)$ of the $n$ alternatives under consideration; the value assigned to $a_{i j}$ is typically in the interval $[1 / 9,9]$. The estimated weight vector $w$ is found by solving the following eigen vector problem:

$$
A w=\lambda_{\max } w
$$

Where $\lambda_{\max }$ is the principle eigenvalue of $A$.

Therefore,

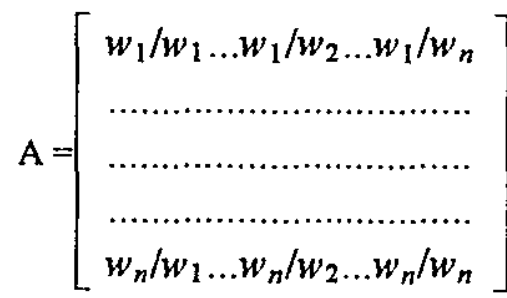

As an example, assume that one is given three machines of different criticality according to their downtime failures. These machines are $a, b$, and $c$ of criticality 3,5 , and 7 hours respectively, taking downtime as a criteria. Suppose that a matrix of pairwise ratios is formed whose rows give the ratios of the downtime of each machine with respect to all others. Thus one has the equation of :

$A w=b\left[\begin{array}{lll}a & b & c \\ 3 / 3 & 3 / 5 & 3 / 7 \\ 5 / 3 & 5 / 5 & 5 / 7 \\ 7 / 3 & 7 / 5 & 7 / 7\end{array}\right]\left[\begin{array}{l}3 \\ 5 \\ 7\end{array}\right]=3\left[\begin{array}{l}3 \\ 5 \\ 7\end{array}\right]=n w \ldots .(3)$

where $A$ has been multiplied on the right by the vector of weights $w$. The result of this multiplication is $n w$. Thus, to recover the scale from the matrix of ratios, it is necessary solve the problem $A w=n w$. This is a system of homogeneous linear equations. It has a nontrivial solution if and only if the determinant of $A-n I$ vanishes, that is, $n$ is an eigenvalue of $A$.

\section{Appendix (2): The Theory Of Fuzzy Logic Controllers (F.L.C.) :}

In this section theory of FLC is presented. In fuzzy set theory:-

$\mu_{A}: X \rightarrow[0,1]$ where $\mu_{A}$, is degree of membership, and $\mathrm{X}$ is the universal set defined in a specific problem. Also, fuzzy set operations:-

The Union of $\mathrm{A}$ and $\mathrm{B}(A \vee B)$ is a fuzxy set with the following membership function:-

$\mu_{A \cup B}=\max \left\{\mu_{A}, \mu_{B}\right\}$

The Intersection of $\mathrm{A}$ and $\mathrm{B}(A \wedge B)$ is a fuzzy set with the following membership function:-

$\mu_{A \cap B}=\min \left\{\mu_{A}, \mu_{B}\right\}$

In the design of a FLC, one must identify the main control parameters and determine a term set which is at the right level of granularity for describing the values of each linguistic values of each linguistic variable. For example, a term set including linguistic values such as \{Low, Medium, Large\} may not be satisfactory in some domains, and may instead require the use of a five term set such as \{Very Low, Low, Medium, Large, and Very Large $\}$. After the values of the main control parameters are determined, a knowledge base is developed using the above control variables and the values that they may take. If the knowledge base is a rule base, more than one rule may fire requiring the selection of a conflict resolution method for decision making, as will be described later. The computation of FLC output is achieved by firstly fuzzifying the crisp input through finding out the intersection of the label's membership function. Then given the rule -base, one can apply the inference rule of maximum of the minimums (Max-Min Composition) using the AND and OR operators.

\section{Compositional Rule of Inference:}

Given a binary relation $R$, in the space $U x V$, then a subset $B^{\prime}$ of $V$ can be inferred from the subset $A^{\prime}$ of $U$ using the compositional rule of inference written as;

$$
B^{\prime}=A^{\prime} \cdot R
$$

where $\mathrm{R}$ is the relation between $\mathrm{A}$ and $\mathrm{B}$, the grade of membership function $\mathrm{B}^{\prime}$ is defined as:

$\mu_{B}(u)=\max \left\{\min \left[\mu_{A}(u) ; \mu_{R}(u, v)\right]\right\}$

where, $u \in U$, and $v \in V$.

This is equivalent to the product of a vector and a matrix with multiplication replaced by "min" and addition by "max" operators. The inferred result will be the maximum of those minimums. This inference rule underlies most of the fuzzy controller theory. 